\title{
Split-field finite-difference time-domain method for second-harmonic generation in two-dimensionally periodic structures
}

\author{
Jorge Francés, ${ }^{1,2, *}$ Jani Tervo, ${ }^{3}$ Sergi Gallego, ${ }^{1,2}$ Sergio Bleda, ${ }^{1,2}$ Cristian Neipp,${ }^{1,2}$ and Andrés Márquez ${ }^{1,2}$ \\ ${ }^{1}$ Department of Physics, Systems Engineering and Signal Theory, University of Alicante, San Vicente del Raspeig Drive, \\ NW, Alicante, Ap. 99, E-3080, Spain \\ ${ }^{2}$ University Institute of Physics Applied to Sciences and Technologies, University of Alicante, San Vicente del Raspeig \\ Drive, NW, Alicante, Ap. 99, E-3080, Spain \\ ${ }^{3}$ Institute of Photonics, University of Eastern Finland, P.O. Box 111, FI-80101 Joensuu, Finland \\ *Corresponding author: jfmonllor@ua.es
}

Received January 13, 2015; revised February 18, 2015; accepted February 18, 2015; posted February 19, 2015 (Doc. ID 232421); published March 20, 2015

\begin{abstract}
The split-field finite-difference time-domain method is extended to second-harmonic generation in twodimensionally periodic structures. Making use of the full coefficient-tensor formalism, a coupled nonlinear system of equations, which must be solved at each update of the electromagnetic field, is developed. The accuracy of the method is verified by comparing the results to well-known one-dimensional problems. The results for L-shaped arrays are compared with results obtained with the Fourier modal method. @ 2015 Optical Society of America OCIS codes: (050.1755) Computational electromagnetic methods; (050.1950) Diffraction gratings;
\end{abstract}

(190.4360) Nonlinear optics, devices. http://dx.doi.org/10.1364/JOSAB.32.000664

\section{INTRODUCTION}

Recently, the applications of nonlinear optics in communications, optical computing, and photonic crystal structures have been extensively investigated in the literature [1-7]. Nonlinear-optical materials reach different phenomena such as all-optical control of electromagnetic waves or all-optical signal processing $[\underline{8}, 9]$. While these effects are usually weak and high amplitudes of the electromagnetic field are often required, nonlinear effects may sometimes be observed with lower input intensities due to field confinement in optical nanostructures [1,4]. For example, second-harmonic generation (SHG) in periodic nanostructures has attracted much interest due to its applications such as the determination of surface magnetic and electric properties [10,11], rough surface studies [12], and apertureless scanning near-field microscopy [13].

The classical finite-difference time-domain (FDTD) method, introduced by Yee in 1966 [14], has been successfully applied to nonlinear media [15-21]. However, it encounters difficulties in analyzing periodic media under oblique incidence. This issue is addressed by the split-field FDTD (SF-FDTD) method [22], which has lately been extended, for example, to one-dimensionally (1D) periodic structures made of anisotropic media [23,24] and to two-dimensionally (2D) periodic components made of dispersive [25,26] or anisotropic [27] materials. Recently, SF-FDTD has also been extended to nonlinear media in two dimensions [28,29]. In particular, the study of Francés et al. [29] showed the extension of the SF-FDTD for the specific case of SHG in a 1D crystal material with cubic symmetry. Such symmetry may be used to simplify the full second-order susceptibility tensor to only three coefficients. This setup provides a decoupling of the pump and second-harmonic fields in terms of polarization, thus simplifying the numerical implementation.

In this paper, we introduce the SF-FDTD approach for analyzing SHG in 2D periodic structures with no restrictions to the material symmetries. We first formulate the nonlinear system of equations to be solved by means of a fixed-point procedure adapted from $[28,29]$. We then validate the method by analyzing SHG under quasi-phase matching (QPM) in homogenous media as well as L-shaped nanostructures (cf. [30]).

\section{EXTENDED SF-FDTD FOR TWO- DIMENSIONALLY MODULATED NONLINEAR MEDIA}

Let us consider the geometry illustrated in Fig. 1 . We assume a micro- or nanostructured region between homogeneous semiinfinite linear, isotropic, and nonmagnetic media. The modulated region is $2 \mathrm{D}$ periodic with periods $\Lambda_{1}$ and $\Lambda_{2}$, and has a thickness $h$.

The incident pump field is a linearly polarized plane wave with a wavevector $\mathbf{k}_{0}$,

$$
\begin{aligned}
\mathbf{k}_{0} & =k_{x} \hat{x}+k_{y} \hat{y}+k_{z} \hat{z} \\
& =\frac{\omega}{c}(\sin \theta \sin \phi \hat{x}+\sin \theta \sin \phi \hat{y}+\cos \theta \hat{z}),
\end{aligned}
$$

where $\theta$ and $\phi$ are the polar and azimuthal angles, respectively. Furthermore, $k_{j}, j=x, y, z$ denote the Cartesian components of the wavevector, $\omega$ is the (angular) frequency, and $c$ is the speed of light. 


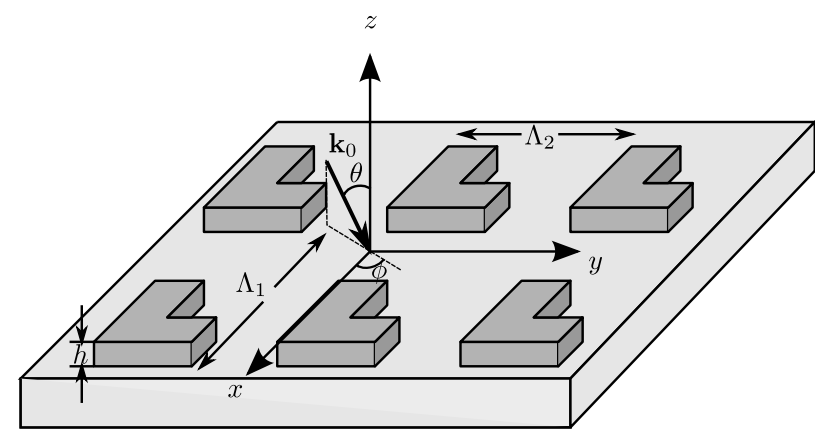

Fig. 1. Schematic illustration of a $2 \mathrm{D}$ periodic structure and the incidence configuration.

\section{A. Fundamentals of the SF-FDTD Method}

Figure 2(a) shows the problem space for a typical SF-FDTD simulation of periodic media. The convention for the spatial arrangement of the electric field $\mathbf{E}$ and the magnetic field $\mathbf{H}$, called the Yee cell, is shown in Fig. 2(b). Such an arrangement simplifies the finite-difference form of the curl operator, and it is one of the main characteristics of FDTD schemes. In this work, we assume a structure that is periodic in the $x$ and $y$ directions, and illuminate the computational grid from a plane parallel to the $x y$ plane for illuminating the grid. Both $z$ boundaries are terminated with perfectly matched layers (PMLs) that eliminate undesired reflections [23,27,31]. The $x y$ boundaries are handled by the periodic boundary conditions (PBCs). In order to accomplish this task successfully, the split-field method uses a set of transformed fields that eliminate the phase difference between the two adjacent sides of the problem space.

In order to simplify the derivation of the new approach presented here, we briefly recall the fundamentals of the derivation of SF-FDTD from earlier studies. Assuming nonmagnetic and nonconducting media, Maxwell's curl equations take on the forms

$$
\begin{gathered}
\nabla \times \mathbf{E}=-j \omega \mu_{0} \mathbf{H}, \\
\nabla \times \mathbf{H}=j \omega \epsilon_{0} \epsilon_{r} \mathbf{E}+j \omega \mathbf{F}^{\mathrm{NL}},
\end{gathered}
$$

where $\epsilon_{0}$ and $\mu_{0}$ are the vacuum permittivity and permeability, respectively, $\epsilon_{r}$ denotes the relative linear permittivity, and $\mathbf{F}^{\mathrm{NL}}$ is the nonlinear polarization.

The SF-FDTD considers a transformation of the electric and magnetic fields taking into account that the new variables implicitly contain the oblique field propagation, which leads to new split-field variables

$$
\mathbf{P}=\mathbf{E} e^{j\left(k_{x} x+k_{y} y\right)},
$$

$$
\mathbf{Q}=c \mu_{0} \mathbf{H} e^{j\left(k_{x} x+k_{y} y\right)},
$$

where $\mathbf{P}$ and $\mathbf{Q}$ are the transformed vectors in the phasor domain. Analogous transformation can also be applied to the nonlinear polarization term [28] by introducing a new transformed vector variable $\mathbf{G}$,

$$
\mathbf{G}^{\mathrm{NL}}=\mu_{0} c \mathbf{F}^{\mathrm{NL}} e^{j\left(k_{x} x+k_{y} y\right)} .
$$

Substituting the split-field components into Maxwell's equations $[27,28]$, the basis for the SF-FDTD with second-order nonlinear polarization terms can be expressed as follows:

$$
\begin{gathered}
\frac{j \omega}{c} \mathbf{P}=\kappa \nabla \times \mathbf{Q}+\frac{j \omega}{c} \kappa \mathbf{q} \mathbf{Q}-j \omega \mathbf{G}^{\mathrm{NL}}, \\
\frac{j \omega}{c} \mathbf{Q}=-\nabla \times \mathbf{P}-\frac{j \omega}{c} \kappa \mathbf{q} \mathbf{P},
\end{gathered}
$$

where $\kappa=\epsilon_{r}^{-1}$ and the matrix $\mathbf{q}$ is defined as (a)

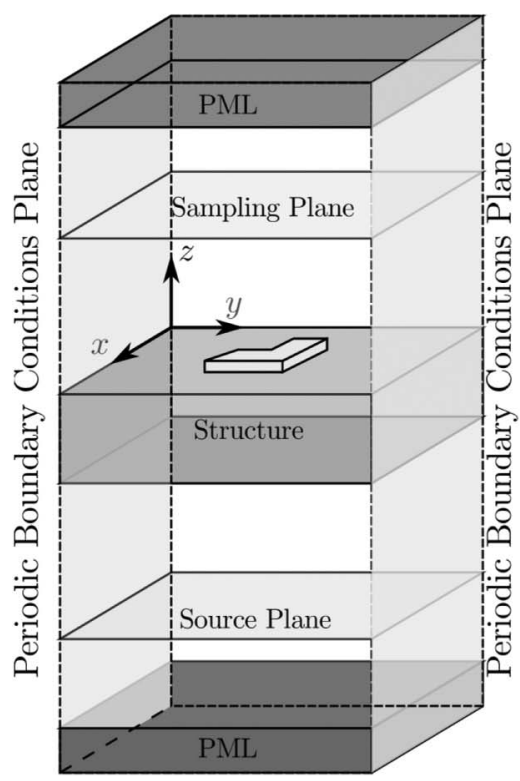

(b)

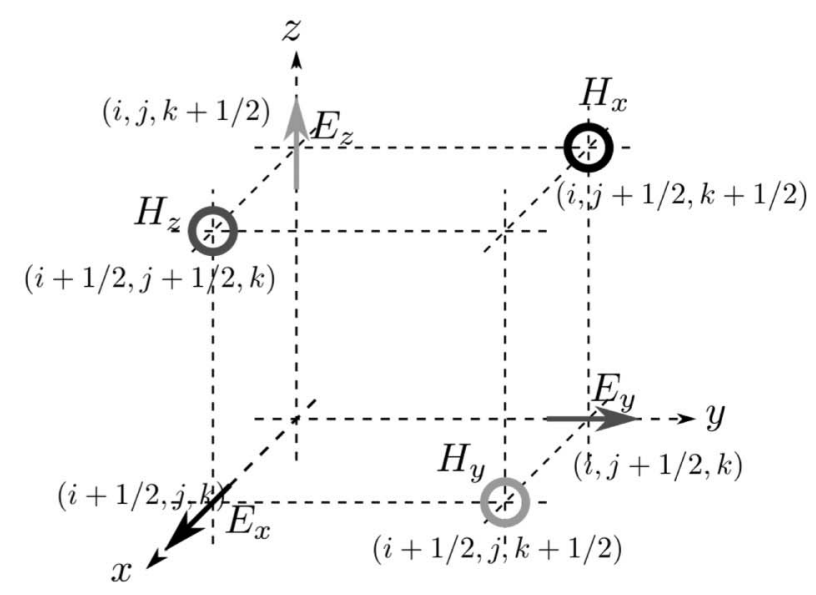

Fig. 2. (a) General problem space for SF-FDTD simulations used in this paper. (b) Yee cell for a three-dimensional FDTD scheme. 


$$
\mathbf{q}=\frac{\omega}{c}\left[\begin{array}{ccc}
0 & 0 & -k_{y} \\
0 & 0 & k_{x} \\
k_{y} & -k_{x} & 0
\end{array}\right] .
$$

The presence of time-derivative terms on each side of Eqs. (7) and (8) makes it difficult to redefine these expressions into finite differences. In order to solve this issue a new set of variables is defined on the right-hand side, separating both $\mathbf{P}$ and $\mathbf{Q}$ into two components:

$$
\begin{gathered}
\mathbf{P}=\mathbf{P}_{a}+\kappa \mathbf{q} \mathbf{Q}-c \mathbf{G}^{\mathrm{NL}}, \\
\mathbf{Q}=\mathbf{Q}_{a}-\mathbf{q} \mathbf{P} .
\end{gathered}
$$

The update-finite-difference expressions for the " $a$ " fields can easily be derived substituting Eqs. (10) and (11) into Eqs. (7) and (8) and discretizing the equations with respect to time, which yields

$$
\begin{gathered}
\frac{1}{c \Delta t}\left[\mathbf{P}_{a}^{n+1}-\mathbf{P}_{a}^{n}\right]=\kappa \nabla \times \mathbf{Q}^{n+1 / 2}, \\
\frac{1}{c \Delta t}\left[\mathbf{Q}_{a}^{n+1}-\mathbf{Q}_{a}^{n}\right]=-\nabla \times \mathbf{P}^{n+1 / 2},
\end{gathered}
$$

where $\Delta t$ is the time resolution, which is related with the spatial resolution by means of the Courant-Friederichs-Lewy condition [32]. The SF-FDTD algorithm is not an unconditionally stable method, and the stability is affected by many parameters such as the CFL condition previously mentioned, additional averaging, and larger angles of incidence. Using the lower bound found by Roden et al. [22] is sufficient to ensure stability in the most common scenarios. Therefore, the CFL condition is chosen small enough in order to ensure stability and convergence to proper solutions. This assumption also implies smaller time and spatial resolutions and hence bigger grid sizes and larger time simulations and computational resources. It is worthwhile to note that finite-difference schemes have an exponentially growing cost in terms of grid size, so enlarging the grid size has a dramatic impact on the time simulation costs. More information regarding the stability, convergence, and computational optimization can be found in [15,22-24,27].

The SF-FDTD leapfrog algorithm used for updating field equations uses Eqs. $(\underline{12})$ and $(\underline{13)}$ to update " $a$ " fields from the $\mathbf{P}$ and $\mathbf{Q}$ fields obtained previously. After that, the current $\mathbf{P}$ field can be calculated by means of

$$
\mathbf{P}=\frac{\mathbf{P}_{a}+\kappa \mathbf{q} \mathbf{Q}_{a}-c \mathbf{G}^{\mathrm{NL}}}{\mathbf{I}+\kappa \mathbf{q}^{2}},
$$

where I is the identity matrix. After $\mathbf{P}$ has been updated, the components of $\mathbf{Q}$ are straightforwardly obtained from Eq. (11). An interested reader can find more details about the derivation of the equations in SF-FDTD in [23,25-27]. In the following section, we discuss the implementation of the polarization terms in Eq. (14) for the specific case of a tensorial second-order nonlinear susceptibility.

\section{B. Formulation of Second-Order Nonlinear Media}

The polarization $\mathbf{F}^{\mathrm{NL}}$ that models the nonlinear effects in noncentrosymmetric media relates the second-order nonlinear susceptibility and the field components inside the structure. The presence of a fundamental or pump field at frequency $\omega_{f}$ leads to an exchange of energy with the second-harmonic field at the double frequency $\omega_{s}=2 \omega_{f}$. The nonlinear polarization term is defined in terms of a third-rank tensor $\mathbf{d}$ that, in the case of SHG, can be represented in contracted form as a $3 \times 6$ matrix $d_{m \xi}[\underline{1}, \underline{29}, \underline{33}]$,

$$
\mathbf{d}=\left[\begin{array}{llllll}
d_{11} & d_{12} & d_{13} & d_{14} & d_{15} & d_{16} \\
d_{21} & d_{22} & d_{23} & d_{24} & d_{25} & d_{26} \\
d_{31} & d_{32} & d_{33} & d_{34} & d_{35} & d_{36}
\end{array}\right],
$$

which is related to the second-order susceptibility tensor by

$$
d_{m \alpha \beta}=\frac{1}{2} \chi_{m \alpha \beta}^{(2)},
$$

and Table 1 that relates the indices of tensors $d_{m \alpha \beta}$ and $d_{m \xi}$. Thus, suffix $\xi$ takes on values $1 . .66$ that are related to the Cartesian axes by the rules given in the table [1,33]. Using this contracted suffix notation, the polarization term related to the SHG can be defined by [33-36]

$$
\begin{gathered}
F_{m}^{\mathrm{NL}, \omega_{f}}=2 \epsilon_{0} \sum_{\sigma, \beta} d_{m \xi} E_{\sigma}^{\omega_{f}} E_{\beta}^{\omega_{s}}, \\
F_{m}^{\mathrm{NL}, \omega_{s}}=\epsilon_{0} \sum_{\sigma, \beta} d_{m \xi} E_{\sigma}^{\omega_{f}} E_{\beta}^{\omega_{f}},
\end{gathered}
$$

where $m=1,2$, and 3 stand for $x, y$, and $z$, respectively.

Taking into account the transformation into the split-field domain we find that

$$
\begin{gathered}
{\left[\begin{array}{c}
G_{x}^{\mathrm{NL}, \omega_{f}} \\
G_{y}^{\mathrm{NL}, \omega_{f}} \\
G_{z}^{\mathrm{NL}, \omega_{f}}
\end{array}\right]=\frac{2}{c} \mathbf{d}\left[\begin{array}{c}
P_{x}^{\omega_{f}} E_{x}^{\omega_{s}} \\
P_{y}^{\omega_{f}} E_{y}^{\omega_{s}} \\
P_{z}^{\omega_{f}} E_{z}^{\omega_{s}} \\
P_{z}^{\omega_{f}} E_{y}^{\omega_{s}}+P_{y}^{\omega_{f}} E_{z}^{\omega_{s}} \\
P_{z}^{\omega_{f}} E_{x}^{\omega_{s}}+P_{x}^{\omega_{f}} E_{z}^{\omega_{s}} \\
P_{x}^{\omega_{f}} E_{y}^{\omega_{s}}+P_{y}^{\omega_{f}} E_{x}^{\omega_{s}}
\end{array}\right],} \\
{\left[\begin{array}{c}
G_{x}^{\mathrm{NL}, \omega_{s}} \\
G_{y}^{\mathrm{NL}, \omega_{s}} \\
G_{z}^{\mathrm{NL}, \omega_{s}}
\end{array}\right]=\frac{1}{c} \mathbf{d}\left[\begin{array}{c}
P_{x}^{\omega_{f}} P_{x}^{\omega_{f}} \\
P_{y}^{\omega_{f}} P_{y}^{\omega_{f}} \\
P_{z}^{\omega_{f}} P_{z}^{\omega_{f}} \\
2 P_{z}^{\omega_{f}} P_{y}^{\omega_{f}} \\
2 P_{z}^{\omega_{f}} P_{x}^{\omega_{f}} \\
2 P_{x}^{\omega_{f}} P_{y}^{\omega_{f}}
\end{array}\right] .}
\end{gathered}
$$

Once the " $a$ " fields are known, we can calculate the total fields. They are found to be expressed using only the " $a$ " fields and the inner electric field components by inserting Eqs. (19) and (20) into Eq. (14), which reveals that

Table 1. Contracted Matrix Notation for $d_{m \xi}$ Indices

\begin{tabular}{ccccccc}
\hline$\xi$ & 1 & 2 & 3 & 4 & 5 & 6 \\
\hline \multirow{2}{*}{$\sigma \beta$} & $x x$ & $y y$ & $z z$ & $z y$ & $z x$ & $x y$ \\
& & & & $y z$ & $x z$ & $y x$ \\
\hline
\end{tabular}




$$
\begin{gathered}
P_{x}^{\omega_{f}}=\frac{P_{x a}^{\omega_{f}}-\kappa\left(k_{y} Q_{z a}^{\omega_{f}}-k_{x} k_{y} P_{y}^{\omega_{f}}-c \bar{G}_{x}^{\mathrm{NL}, \omega_{f}}\right)}{1+\kappa\left[k_{y}^{2}+2\left(d_{11} E_{x}^{\omega_{s}}+d_{15} E_{z}^{\omega_{s}}+d_{16} E_{y}^{\omega_{s}}\right)\right]}, \\
P_{y}^{\omega_{f}}=\frac{P_{y a}^{\omega_{f}}+\kappa\left(k_{x} Q_{z a}^{\omega_{f}}+k_{x} k_{y} P_{x}^{\omega_{f}}-c \bar{G}_{y}^{\mathrm{NL}, \omega_{f}}\right)}{1+\kappa\left[k_{x}^{2}+2\left(d_{22} E_{y}^{\omega_{s}}+d_{24} E_{z}^{\omega_{s}}+d_{26} E_{x}^{\omega_{s}}\right)\right]}, \\
P_{z}^{\omega_{f}}=\frac{P_{z a}^{\omega_{f}}+\kappa\left(k_{y} Q_{x a}^{\omega_{f}}-k_{x} Q_{y a}^{\omega_{f}}-c \bar{G}_{z}^{\mathrm{NL}, \omega_{f}}\right)}{1-\kappa\left[k_{x}^{2}+k_{y}^{2}-2\left(d_{33} E_{z}^{\omega_{s}}+d_{34} E_{y}^{\omega_{s}}+d_{35} E_{x}^{\omega_{s}}\right)\right]}, \\
P_{x}^{\omega_{s}}=\frac{P_{x a}^{\omega_{s}}-\kappa\left(k_{y} Q_{z a}^{\omega_{s}}-k_{x} k_{y} P_{y}^{\omega_{s}}-c G_{x}^{\mathrm{NL}, \omega_{s}}\right)}{1+\kappa k_{y}^{2}}, \\
P_{y}^{\omega_{s}}=\frac{P_{y a}^{\omega_{s}}+\kappa\left(k_{x} Q_{z a}^{\omega_{s}}+k_{x} k_{y} P_{x}^{\omega_{s}}-c G_{y}^{\mathrm{NL}, \omega_{s}}\right)}{1+\kappa k_{x}^{2}}, \\
P_{z}^{\omega_{s}}=\frac{P_{z a}^{\omega_{s}}+\kappa\left(k_{y} Q_{x a}^{\omega_{s}}-k_{x} Q_{y a}^{\omega_{s}}-c G_{z}^{\mathrm{NL}, \omega_{s}}\right)}{1-\kappa\left(k_{x}^{2}+k_{y}^{2}\right)},
\end{gathered}
$$

where $\overline{\mathbf{G}}^{\mathrm{NL}, \omega_{f}}$ is defined as

$$
\begin{aligned}
& \bar{G}_{x}^{\mathrm{NL}, \omega_{f}}=\frac{2}{c}\left[d_{12} P_{y}^{\omega_{f}} E_{y}^{\omega_{s}}+d_{13} P_{z}^{\omega_{f}} E_{z}^{\omega_{s}}+d_{14}\left(P_{z}^{\omega_{f}} E_{y}^{\omega_{s}}\right.\right. \\
&\left.\left.+P_{y}^{\omega_{f}} E_{z}^{\omega_{s}}\right)+d_{15} P_{z}^{\omega_{f}} E_{x}^{\omega_{s}}+d_{16} P_{y}^{\omega_{f}} E_{x}^{\omega_{s}}\right], \\
& \bar{G}_{y}^{\mathrm{NL}, \omega_{f}}=\frac{2}{c}\left[d_{21} P_{x}^{\omega_{f}} E_{x}^{\omega_{s}}+d_{23} P_{z}^{\omega_{f}} E_{z}^{\omega_{s}}+d_{24} P_{z}^{\omega_{f}} E_{y}^{\omega_{s}}\right. \\
&\left.+d_{25}\left(P_{z}^{\omega_{f}} E_{x}^{\omega_{s}}+P_{x}^{\omega_{f}} E_{z}^{\omega_{s}}\right)+d_{26} P_{x}^{\omega_{f}} E_{y}^{\omega_{s}}\right], \\
& \bar{G}_{z}^{\mathrm{NL}, \omega_{f}}= \frac{2}{c}\left[d_{31} P_{x}^{\omega_{f}} E_{x}^{\omega_{s}}+d_{32} P_{y}^{\omega_{f}} E_{y}^{\omega_{s}}+d_{34} P_{y}^{\omega_{f}} E_{z}^{\omega_{s}}\right. \\
&\left.+d_{35} P_{x}^{\omega_{f}} E_{z}^{\omega_{s}}+d_{36}\left(P_{x}^{\omega_{f}} E_{y}^{\omega_{s}}+P_{y}^{\omega_{f}} E_{x}^{\omega_{s}}\right)\right] .
\end{aligned}
$$

Due to the staggered spatial arrangement of the electromagnetic field an averaging over the nearest nodes surrounding each component field is required. For example, in Eq. (21), the values of $P_{y}^{\omega_{f}}, E_{z}^{\omega_{s}}$, and $E_{y}^{\omega_{s}}$ at the $P_{x}^{\omega_{f}}$ position $(i+\overline{1 / 2}$, $j, k)$ must be computed since they are defined in different spatial positions inside the Yee cell [see Fig. 2(b)]. Assuming that field components are simply the arithmetic average of the stored values in the neighboring cells, the semi-implicit approximation can be applied in order to address this issue $[\underline{23}, \underline{29}, \underline{37}]$.

A nonlinear system of equations of the form $\mathbf{P}=\mathbf{U}(\mathbf{P})$ is made up by Eqs. (21)-(26). For solving this system of equations a fixed-point iterative process is considered. The basis of this method is solving the following iterative process with the form

$$
\mathbf{P}^{(p+1)}=\mathbf{U}\left(\mathbf{P}^{(p)}\right), \quad p=0,1,2, \ldots,
$$

where $p$ is for the iterations of the fixed-point procedure.

It is worth noting that the fixed-point process must be performed at each time step of the FDTD simulation. The scheme is based on considering an initial guess of $\mathbf{P}$, which represents

those fields obtained from considering linear media, after which subsequent iterations are carried out to improve the accuracy of the results with every iteration. As mentioned in [28], 30 steps have been chosen for the fixed-point iterative process since it provided good results near the upper limit of convergence of the method. The amplitude of the electric field $E$ has to be limited to an upper value that is related to the amplitude of the second-order susceptibility to assure convergence of the fixed-point iteration. The authors have concluded experimentally that using Eq. (54) in [28] substituting the third-order susceptibility contribution by means of the highest second-order susceptibility an upper limit of the pump incident field is provided. More details regarding the convergence condition for the fixed-point iterative process and FDTD can be found in $[\underline{28}, \underline{38}]$.

\section{NUMERICAL EXAMPLES}

In order to demonstrate the accuracy of the method presented here, two numerical examples are given. First, a 1D periodic GaAs-based homogeneous layer is considered. This example has been simulated previously with the 2D SF-FDTD scheme [29], and it is a well known SHG scenario, in which the phase matching of the second-harmonic field can be easily analyzed. Second, we show the analysis of a 2D L-shaped particle array that has been investigated experimentally and numerically by means of the Fourier modal method (FMM) [30,39-41] and the boundary element method [42]. Here, however, we study dielectric nanostructures only.

\section{A. Quasi-Phase Matching in a Homogeneous Layer}

The GaAs-based homogeneous layer is illuminated by means of continuous-wave TM signal at $2.25 \mu \mathrm{m}$, intensity $120 \mu \mathrm{W} / \mathrm{m}^{2}$, and angle of incidence $30^{\circ}$. The physical parameters of this material are fully defined in Section 3.B and [1]. Two different situations are considered to observe the SHG. The first is a no-matching technique, and the variation of the amplitude for the second-harmonic field can be easily identified due to the phase shift between the pump and the secondharmonic field. The second considers the QPM technique implemented by alternately inverting the orientation of the crystal axes along the material. The optimum period for the QPM structure is given by $\Delta=2 L_{\text {coh }}$, with $L_{\text {coh }}=7.1 \mu \mathrm{m}$ for SHG of radiation at a wavelength of $1.25 \mu \mathrm{m}$ at the oblique angle of incidence in AsGa. If the period of the alteration of the axis is close to twice the coherent buildup length of the nonlinear interaction, the interchange between the fundamental field and the second-harmonic field tends to grow along the nonlinear media. Without QPM there is a periodic variation of the second-harmonic amplitude as a function of the space. Both phenomena are represented in Fig. 3 .

\section{B. Second-Harmonic Generation by L-Shaped Nanoparticles}

Our next example deals with an array of L-shaped nanoparticles made of GaAs $\left(n_{0}=3.47\right)$ [1]. Figures 1 and $\underline{4}$ show the geometry considered in this case. Each $\overline{\mathrm{L}}$ particle has two arms of length $l=150 \mathrm{~nm}$ and width $w=80 \mathrm{~nm}$ and is arranged with periods $\Lambda_{1}=\Lambda_{2}=400 \mathrm{~nm}$. The substrate is fused silica, and the modulated region has a thickness of $h=20 \mathrm{~nm}$. 


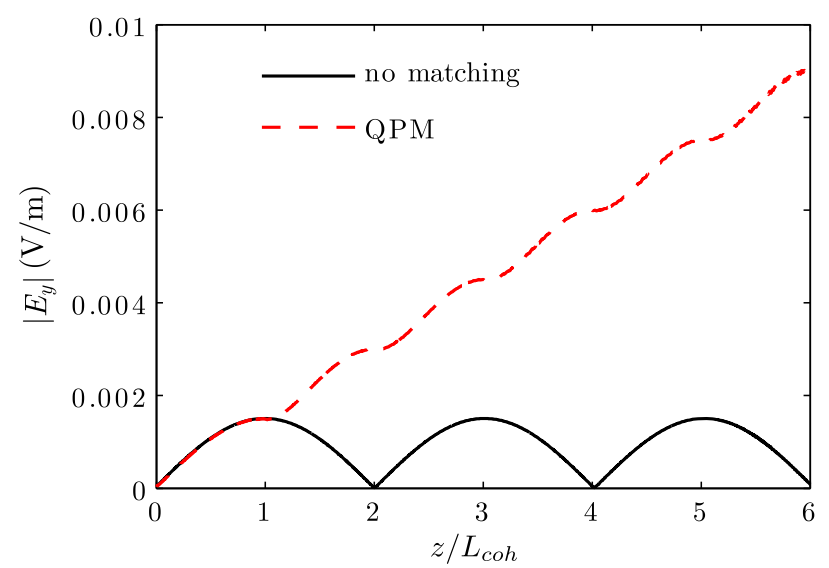

Fig. 3. Amplitude of the second-harmonic field along the nonlinear material (solid line, no matching; dashed line, QPM).

Gallium arsenide is classified as a crystal point group $\overline{4} 3 \mathrm{~m}$. In this case, the only nonzero elements in the nonlinearoptical coefficient tensor are $d_{14}=d_{25}=d_{36}=370 \mathrm{pm} / \mathrm{V}$ [1] . The crystal axes are taken following the same strategy in [30]; thus the crystal axes match the $A$ axes in Fig. 4 . In order to fit these parameters into the SF-FDTD equations a coordinate transformation has to be made so as to get the values of the susceptibility tensor in the $O_{x y z}$ system.

The $(0,0)$ th-order transmitted SHG intensity as a function of the input pump intensity for both type-I and type-II samples is calculated by means of the SF-FDTD method. Directions $A, B$, and $C$ are the polarization directions of normally incident plane waves with wavelength $1060 \mathrm{~nm}$. The SF-FDTD setup considered is based on a grid of $40 \times 40 \times 300$ cells with spatial and time resolutions of $10 \mathrm{~nm}$ and $1.11 \times 10^{-11} \mathrm{~ns}$, respectively. The number of time steps has been chosen in order to ensure steady-state condition, thus being 7000 time steps.

Figure 5 shows the simulation results of the transmitted SHG intensity of the L-shaped structures. Comparing it with the results obtained by Bai and Turunen [30] with FMM, we can see that although they are not perfectly coincident, many characteristics are well reproduced if we take into account that we are not using metallic L-shaped nanostructures. Nevertheless, the SHG output intensity has an expected quadratic relation with the input intensity; the stronger output of SHG for polarizations $B$ and $C$ for the type-I structure is also corroborated. However, there are some differences between the results obtained here and those derived from the FMM and metallic nanoparticles. These differences would basically be produced by the enhancement produced by metallic

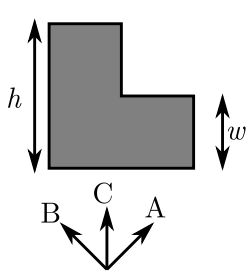

(a)

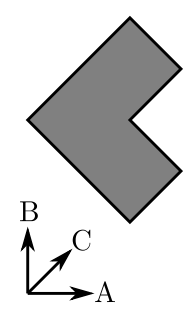

(b)
Fig. 4. Top view of (a) type-I and (b) type-II L-particle arrays with two equal arms with length $l$ and width $w$.

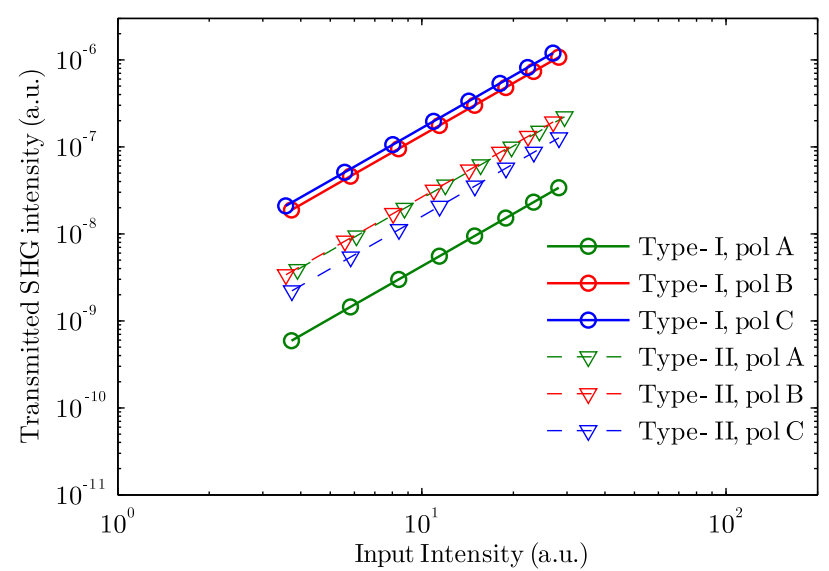

Fig. 5. Simulation of the $(0,0)$ th-order transmitted SHG intensity as a function of the input intensity for both type-I and type-II structures and for polarization directions $A, B$, and $C$. The results are comparable with those shown in Fig. 6 in [30].

nanostructures of the electromagnetic field. Even considering the differences between the nonlinear behavior found in metallic nanoparticles and that found in AsGa-based nanopartices, the authors found that the results presented here are consistent with those obtained in [30,39], since many characteristics are common in both scenarios. The accurate analysis of the metallic nanoparticles is beyond the scope of this paper and will be addressed in the near future.

\section{CONCLUSIONS}

An extension of the SF-FDTD method for the analysis of SHG in $2 \mathrm{D}$ periodic structures with nonlinear materials and an arbitrary susceptibility tensor is defined. This method takes no hypothesis, and $\mathrm{SH}$ and pump fields are solved by means of a fixed-point iterative process. The reliability of the method is demonstrated by means of the analysis of a SHG case based on a 1D nonlinear material with and without QPM. Then it is used to simulate the $\mathrm{SH}$ response of the L-shaped nanoparticle arrays. The results obtained from this analysis are compared with those of the previous analysis carried out with FMM, showing a high degree of consistency. The authors are currently working on including some formalisms for accurately simulating metal structures and also different strategies in order to reduce the computational costs.

\section{ACKNOWLEDGMENTS}

This work is partially supported by the "Ministerio de Economía y Competitividad" of Spain under project FIS2011-29803C02-01; by the "Generalitat Valenciana" of Spain under projects PROMETEO/2011/021, ISIC/2012/013, and GV/2014/ 076; and by the "Universidad de Alicante" of Spain under project GRE12-14.

\section{REFERENCES}

1. R. W. Boyd, Nonlinear Optics, 3rd ed. (Academic, 2008).

2. G. P. Agrawal, Nonlinear Fiber Optics, 5th ed. (Academic, 2012).

3. C. Reinke, A. Jafarpour, B. Momeni, M. Soltani, S. Khorasani, A. Adibi, Y. Xu, and R. K. Lee, "Nonlinear finite-difference timedomain method for the simulation of anisotropic, $\chi^{(2)}$, and $\chi^{(3)}$ optical effects," J. Lightwave Technol. 24, 624-634 (2006). 
4. M. Qasymeh, M. Cada, and S. A. Ponomarenko, "Quadratic electro-optic Kerr effect: applications to photonic devices," IEEE J. Quantum Electron. 44, 740-746 (2008).

5. F. Zhou, Y. Liu, Z.-Y. Li, and Y. Xia, "Analytical model for optical bistability in nonlinear metal nano-antennae involving Kerr materials," Opt. Express. 18, 13337-13344 (2010).

6. J. Yuan and J. Yang, "Computational design for efficient secondharmonic generation in nonlinear photonic crystals,” J. Opt. Soc. Am. B 30, 205-210 (2013)

7. L.-C. Zhao and J. Liu, "Localized nonlinear waves in a two-mode nonlinear fiber,” J. Opt. Soc. Am. B 29, 3119-3127 (2012).

8. W. D. Deering and M. I. Molina, "Power switching in hybrid coherent couplers," IEEE J. Quantum Electron. 33, 336-340 (1997).

9. Y. Wang, "Nonlinear optical limiter and digital optical switch by cascaded nonlinear couplers: analysis," J. Lightwave Technol. 17, 292-297 (1999)

10. S. I. Bozhevolny, K. Pedersen, T. Skettrup, X. Zhang, and M Belmonte, "Far- and near-field second-harmonic imaging of ferroelectric domain walls," Opt. Commun. 152, 221-224 (1998).

11. I. I. Smolyaninov, H. Y. Liang, C. H. Lee, C. C. Davis, V. Nagarajan, and R. Ramesh, "Near-field second harmonic imaging of the $\mathrm{c} / \mathrm{a} / \mathrm{c} /$ a polydomain films structure of epitaxial pbzr $\mathrm{ti}_{1-\mathrm{x}} \mathrm{O}_{3}$ thin films," J. Microsc. 202, 250-254 (2001).

12. A. V. Zayats, T. Kalkbrenner, V. Sandoghdar, and J. Mlynek, "Second harmonic generation from individual surface defects under local excitation,” Phys. Rev. B 61, 4545-4548 (2000).

13. S. Takahashi and A. V. Zayats, "Near-field second-harmonic generation at a metal tip apex," Appl. Phys. Lett 80, 3479-3481 (2002).

14. K. S. Yee, "Numerical solution of initial boundary value problems involving Maxwell's equations in isotropic media," IEEE Trans. Antennas Propag. 14, 302-307 (1966).

15. A. Taflove and S. C. Hagness, Computational Electrodynamics: The Finite-Difference Time-Domain Method, 3rd ed. (Artech House, 2005)

16. R. M. Joseph and A. Taflove, "FDTD Maxwell's equations models for nonlinear electrodynamics and optics," IEEE Trans. Antennas Propag. 45, 364-374 (1997).

17. P. M. Goorjian, A. Taflove, R. M. Joseph, and S. C. Hagness, "Computational modeling of femtosecond optical solitons from Maxwell's equations,” IEEE J. Quantum Electron. 28, 2416-2422 (1992).

18. P. M. Goorjian and A. Taflove, "Direct time integration of Maxwell's equations in nonlinear dispersive media for propagation and scattering of femtosecond electromagnetic solitons," Opt. Lett. 17, 180-182 (1992)

19. K. H. Lee, I. Ahmed, R. S. M. Goh, E. H. Khoo, E. P. Li, and T. G. G. Hung, "Implementation of the FDTD method based on Lorentz-Drude dispersive model on GPU for plasmonics applications," Prog. Electromagn. Res. 116, 441-456 (2011).

20. Y.-Q. Zhang and D. B. Ge, "A unified FDTD approach for electromagnetic analysis of dispersive objects," Prog. Electromagn. Res. 96, 155-172 (2009).

21. M. Fujii, M. Tahara, I. Sakagami, W. Freude, and P. Russer, "High-order FDTD and auxiliary differential equation formulation of optical pulse propagation," IEEE J. Quantum Electron. 40, 175-182 (2004).

22. J. Roden, S. D. Gedney, M. P. Kesler, J. G. Maloney, and P. H. Harms, "Time-domain analysis of periodic structures at oblique incidence: orthogonal and nonorthogonal FDTD implementations," IEEE Trans. Microw. Theory Tech. 46, 420-427 (1998).

23. C. Oh and M. J. Escuti, "Time-domain analysis of periodic anisotropic media at oblique incidence: an efficient FDTD implementation," Opt. Express 14, 11870-11884 (2006).

24. J. Francés, S. Bleda, M. L. Álvarez, F. J. Martínez, A. Márquez, C. Neipp, and A. Beléndez, "Acceleration of split-field finite difference time-domain method for anisotropic media by means of graphics processing unit computing," Opt. Eng. 53, 011005 (2013).

25. A. Shahmansouri and B. Rashidian, "Comprehensive threedimensional split-field finite-difference time-domain method for analysis of periodic plasmonic nanostructures: near- and far-field formulation,” J. Opt. Soc. Am. B 28, 2690-2700 (2011).

26. A. Shahmansouri and B. Rashidian, "GPU implementation of split-field finite-difference time-domain method for DrudeLorentz dispersive media,” Prog. Electromagn. Res. 125, 55-77 (2012).

27. M. N. Miskiewicz, P. T. Bowen, and M. J. Escuti, "Efficient 3D FDTD analysis of arbitrary birefringent and dichroic media with obliquely incident sources," Proc. SPIE 8255, 82550W (2012).

28. J. Francés, J. Tervo, and C. Neipp, "Split-field finite-difference time-domain scheme for Kerr-type nonlinear periodic media," Prog. Electromagn. Res. 134, 559-579 (2013).

29. J. Francés, J. Tervo, and A. Márquez, "Tensorial split-field finitedifference time-domain approach for second- and third-order nonlinear materials,” J. Opt. Soc. Am. B 30, 1711-1719 (2013).

30. B. Bai and J. Turunen, "Fourier modal method for the analysis of second-harmonic generation in two-dimensionally periodic structures containing anisotropic materials," J. Opt. Soc. Am. B 24, 1105-1112 (2007).

31. S. D. Gedney, "An anisotropic perfectly matched layer-absorbing medium for the truncation of FDTD lattices," IEEE Trans. Antennas Propag. 44, 1630-1639 (1996).

32. A. Taflove, A. Oskooi, and S. G. Johnson, eds., Advances in FDTD Computational Electrodynamics: Photonics and Nanotechnology (Artech House, 2013).

33. P. Butcher and D. Cotter, The Elements of Nonlinear Optics (Cambrdige University, 1990).

34. H. M. Al-Mudhaffar, M. A. Alsunaidi, and H. M. Masoudi, "Fullwave solution of the second harmonic generation problem using a nonlinear FDTD algorithm," in PIERS Proceedings, Prague, Czech Republic, August 27-30, 2007, pp. 479-482.

35. M. A. Alsunaidi, H. M. Al-Mudhaffar, and H. M. Masoudi, "Vectorial FDTD technique for the analysis of optical secondharmonic generation,” IEEE Photon. Technol. Lett. 21, 310-312 (2009).

36. M. A. Alsunaidi and F. S. Al-Hajiri, "Efficient NL-FDTD solution schemes for the phase-sensitive second harmonic generation problem," J. Lightwave Technol. 27, 4964-4969 (2009).

37. C. Dissanayake, M. Premaratne, I. D. Rukhlenko, and G. P. Agrawal, "FDTD modeling of anisotropic nonlinear optical phenomena in silicon waveguides," Opt. Express 18, 21427-21448 (2010)

38. M. Ammann, "Non-trivial materials in EM-FDTD," Master's thesis (Department of Physics, Swiss Federal Institute of Technology, 2007).

39. H. Tuovinen, J. Turunen, V. Tkachenko, and H. Lemmetynen, "Linear and second-order nonlinear optical properties of arrays of noncentrosymmetric gold nanoparticles," J. Nonlinear Opt. Phys. Mater. 11, 421-432 (2002).

40. B. Canfield, S. Kujala, K. Jefimovs, J. Turunen, and M. Kauranen, "Linear and nonlinear optical responses influenced by broken symmetry in an array of gold nanoparticles," Opt. Express 12, 5418-5423 (2004).

41. B. K. Canfield, S. Kujala, K. Jefimovs, T. Vallius, J. Turunen, and M. Kauranen, "Polarization effects in the linear and nonlinear optical responses of gold nanoparticle arrays," J. Opt. A 7, S110-S117 (2005).

42. J. Mäkitalo, S. Suuriniemi, and M. Kauranen, "Boundary element method for surface nonlinear optics of nanoparticles," Opt. Express 19, 23386-23399 (2011). 